\title{
Pharmacist-led interdisciplinary medication reconciliation using comprehensive medication review in gynaecological oncology patients: a prospective study
}

\author{
Heeyoun Son, ${ }^{1,2}$ Jeongmee $\mathrm{Kim}_{,}{ }^{2}$ Caroline Kim, ${ }^{3}$ Jonathan $\mathrm{Ju}_{1}{ }^{4}$ Youngmee Lee, ${ }^{2}$ \\ Sandy Jeong Rhie ${ }^{5}$
}

${ }^{1}$ Graduate School of Clinical Health Sciences, Ewha Womans University, Seoul, Republic of Korea

2Department of Pharmacy, Samsung Medical Center, Seoul, Republic of Korea ${ }^{3}$ School of Pharmacy, University of Maryland, Baltimore, Maryland, USA

${ }^{4}$ Albany College of Pharmacy and Health Sciences, Albany, New York, USA

${ }^{5}$ College of Pharmacy \& Division of Life and Pharmaceutical Sciences and Graduate School of Clinical Health Sciences, Ewha Womans University, Seoul, Republic of Korea

\section{Correspondence to}

Professor Sandy Jeong Rhie, College of Pharmacy \& Division of Life and Pharmaceutical Sceinces and Graduate School of Clinical Health Sciences, Ewha Womans University, 52 ewhayeodae-gil, Seodaemungu, Seoul 03760, Republic of Korea; sandy.rhie@ewha.ac.kr

Received 10 March 2016 Revised 16 September 2016 Accepted 28 November 2016 Published Online First 20 December 2016

EAHP Statement 4: Clinical Pharmacy Services. EAHP Statement 5: Patient Safety and Quality Assurance

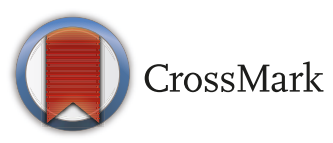

To cite: Son H, Kim J, Kim C, et al. Eur J Hosp Pharm 2018:25:21-25.

\section{ABSTRACT}

Objectives Medication reconciliation is a key part of transitional care. This study examined the implementation of a pharmacist-led medication reconciliation programme for short-term hospitalised patients and explored the barriers and benefits. Methods A prospective study was conducted in patients admitted to a gynaecological oncology department. Medications were reconciled on admission using a 'comprehensive medication review (CMR)' strategy. Patients received a reminder text message and were asked to bring their medications a day before admission for scheduled chemotherapy. Upon admission, a pharmacist reviewed patients' admission prescriptions and home medications, including non-prescription medications, based on clinical status and laboratory test results. Drug-related problems and unused or expired medications were assessed. Satisfaction with the CMR service and reasons for non-compliance were surveyed by an individual interview. The cost of the unused or expired medications was calculated based on the average drug acquisition cost.

Results Sixty-four interventions in 95 patients were performed during the study-namely, correction of treatment duration (34 cases, 53.1\%), recommendation of medications for untreated indications (18 cases, $28.1 \%)$, correct drug selection (5 cases, $7.8 \%$ ), discontinuation of duplicate medications (4 cases, $6.3 \%)$, correction of dose, provision of alternatives for drug-drug interactions, unintended omissions (1 case each, $1.6 \%$ ). The difference in the cost of unused or expired drugs before and after programme implementation was about US\$1700.

Conclusions Pharmacist-led medication reconciliation targeting short-term hospitalised patients improved drug use, prevented medication waste and reduced healthcare costs.

\section{INTRODUCTION}

Medication reconciliation has become a standard activity of pharmacists' practice in the past few decades. ${ }^{1}$ Pharmacist-led medication reconciliation using comprehensive medication review (CMR) has been used to assess allergies, adverse reactions, contraindications, treatment duplication, drug-drug or drug-disease interactions, dosage, or treatment duration, and abuse and misuse of medications. ${ }^{2}$ It has been developed mainly for outpatients in long-term care to optimise treatment, reduce medication errors and reduce the cost of unused medications. Similar programmes, for which outcomes have been reported, have been followed in Denmark, Canada and England. ${ }^{2}$

Drug waste from chronic disease states is a growing concern, particularly with the increasing prevalence of combination drug therapies. Management of maintenance medications for chronic diseases is a fragmented process, and emphasis must be placed on compliance to optimise treatment and reduce drug waste. Drug waste, improper storage and disposal of unused medications may lead to accidental ingestion and medication-related accidents. Furthermore, incorrect disposal of unused medications may pose a serious threat to public health and to the environment. ${ }^{3}$ Despite efforts to reduce drug waste and improve proper prescription drug use, including "Collection and Treatment of Waste Pharmaceuticals in the Household" sponsored by the Korean Ministry of Environment, challenges and barriers remain. Medication reconciliation has been found to reduce changes in drug treatment, and lead to reduction of drug waste when performed routinely. A previous study showed that drug waste from prescription medications is significantly higher than for over-the-counter (OTC) medications, possibly because they are prescribed for a longer period (commonly for 3 months), excessive prescribing of 'as needed' medications and the short expiry date of liquid prescription medications in Korea. ${ }^{4}$ In the UK, a number of strategies have been identified in efforts to reduce drug waste, including deprescribing, polypharmacy monitoring and medication optimisation through intervention and consultation. ${ }^{5}$

The gynaecological oncology service provides high-quality advanced treatment for disorders such as ovarian, endometrial, cervical vulvar and vaginal cancers for women of all ages. The pharmacy service supports the management of chemotherapy, periprocedures, or surgery, and palliative care for advanced or relapsed gynaecological cancer. Patients in a gynaecological oncology department are ideal candidates for CMR, because they have scheduled admission every few weeks for chemotherapy, and these patients tend to have a high interest in their medication management and their overall health.

We implemented a medication reconciliation programme in a gynaecological oncology unit, and conducted CMR and prospective drug use review from preadmission through to discharge. The 
purpose of this study was to improve the safety of medication use, minimise the impact of waste from unused or expired medication and further reduce medication costs by establishing effective pharmacist-directed medication reconciliation with pharmacy interventions.

\section{METHODS}

\section{Subject and data collection}

A prospective interventional study was performed from March to June 2015. Inclusion criteria included patients admitted to a gynaecological oncology unit in a 2000-bed top tier university hospital. Patients aged $<19$ years or who did not agree to participate in the study were excluded. CMR was offered from April to June 2015 and the results were compared with data from March (control). Patient demographics included age, cancer type, comorbidity and discharge placements.

\section{Medication reconciliation process}

A medication reconciliation programme was proposed and agreed with attending physicians and a nurse manager in the gynaecological oncology department. Study subjects were patients with chemotherapy appointments in the computerised physician order entry system. A text message was sent to patients a day before their appointment reminding them to bring their medications. Medication reconciliation was conducted by a gynaecological oncology unit pharmacist or a pharmacy practice resident within 24 hours of admission or on the following working day. Pharmacists reviewed patients' medications using patient medical records and reconciled admission orders and home medications, including prescriptions from other providers, OTC medications, vitamins and herbal and dietary supplements. Unused medications were defined as those which patients had not used owing to non-compliance or that had expired. The pharmacist documented the names and quantity of medications and other pertinent information mentioned by medical team. Patient counselling was then provided. Drug-related problems of the medications, such as indications, dosage, duplication and drug interactions, were assessed. If the medications were allowed to be continued, they were returned to the patients and the physicians were informed. When medications that had expired were found, they were discarded at the hospital.

Drug-related problems, as defined by the American Society of Hospital Pharmacists, were applied to assess patients' medication. The following factors were considered: current admission orders, past medical history and clinical signs and symptoms, and classification of laboratory results, medication without indication, untreated indications, improper drug selection, inappropriate dose, dosage form, treatment duration required to complete the chemotherapy regimen or compliance with treatment protocols, route of administration, duplication, allergy, drug interactions, adverse reactions and unintended omissions. ${ }^{6}$ National Comprehensive Cancer Network Guidelines, Health Insurance Review and Assessment antiemetic guidelines and the Lexicomp Online database were mainly used as references during medication reconciliation.

\section{Outcomes of medication reconciliation}

The amount of unused medications was compared between March (pre-programme implementation) and May (postprogramme implementation). Pill counts, self-report and medication order record of previous discharges were used to verify patient medications and reasons for non-compliance. The WHO Anatomical Therapeutic Chemical classification system was used to categorise patients' unused medications brought to the hospital. Compliance was expressed as a percentage by comparing the quantity of leftover medication subtracted from the dispensed medications with the quantity of dispensed medications; this was the medication possession rate.

Cost analyses were conducted from an institutional perspective and therefore medication acquisition costs were used to determine the value of implementing this service. Cost-benefit was estimated briefly through both cost savings related to reducing drug waste by deprescribing medications at discharge and cost avoidance related to interventions based on the potential to avoid an adverse drug event. We used the probability of harm of 0.3 , as reported by Bates et al, to account for the potential of a particular intervention to prevent harm. ${ }^{7-9}$ There is no cost avoidance report in Korea for adverse drug reaction prevention by pharmaceutical interventions, and thus each intervention was assumed to prevent one day of hospitalisation. The cost of the service was estimated from the pharmacists' salary, corresponding to the time they spent in medication reconciliation.

\section{Patient satisfaction}

One-on-one patient interview was conducted to assess patients' satisfaction with the medication reconciliation programme with CMR. The survey also included patients' willingness to pay for the programme. In addition, we provided counselling based on collected information about patient-reported adverse drug reactions, discharge plan, unexpected medical care, reason for readmissions if any, and patients' knowledge of the medications, providing directions for use, dose, frequency and treatment benefit.

\section{Data analysis}

The number of medication reconciliations, the extent of intervention, amount of drug unused and the estimated reduction in drug waste were calculated. The estimated reduction of unused drugs was compared between the pre- and post-programme using Microsoft Excel 2003. The cost of the drug was calculated based on average drug acquisition costs, and converted into US dollars. Patient feedback was also analysed in order to improve the medication reconciliation programme. This research was approved by the institutional review board at Samsung Seoul Medical Center. (IRB file No. 2015-05-006-01)

\section{RESULTS}

A total of 95 patients were included in the study. All eligible patients were female and the average age was $53.3 \pm 12.0$. The majority of patients had ovarian cancer $(n=66,69.5 \%)$. About $70.5 \%(n=67)$ of patients were discharged to home and $23.2 \%$ $(n=22)$ went to care facilities (table 1$)$.

\section{Medication reconciliation}

An average of nine medications was dispensed per patient at the time of discharge from the previous admission. Pharmacists made 64 interventions throughout the medication reconciliation and $63(98 \%)$ of them were accepted by physicians. Interventions included treatment duration (34 cases, 53.1\%), untreated indications (18 cases, 28.1\%), drug selection (5 cases, $7.8 \%$ ), duplicate therapies (4 cases, 6.3\%), and unintended omission, drug interactions and dose changes (1 case each, 1.6\%) (table 2). Examples of interventions and resolutions are listed in table 3.

Among 95 enrolled patients, 56 patients returned 5946 unused pills. The greatest number of unused medications was for alimentary tract and metabolism (47.4\%), followed by 
Table 1 Demographics $(n=95)$

\begin{tabular}{|c|c|}
\hline Variables & n (\%) \\
\hline \multicolumn{2}{|l|}{ Age (years) } \\
\hline $20-29$ & $1(1.1)$ \\
\hline $30-39$ & $5(5.3)$ \\
\hline $40-49$ & $22(23.2)$ \\
\hline $50-59$ & $34(35.8)$ \\
\hline $60-69$ & $30(31.6)$ \\
\hline $70-79$ & $3(3.2)$ \\
\hline \multicolumn{2}{|l|}{ Cancer type } \\
\hline Ovarian cancer & $66(69.5)$ \\
\hline Cervical cancer & $11(11.6)$ \\
\hline Endometrial cancer & $9(9.5)$ \\
\hline Peritoneal cancer & $2(2.1)$ \\
\hline Fallopian tube cancer & $2(2.1)$ \\
\hline Other cancer & $5(5.3)$ \\
\hline \multicolumn{2}{|l|}{ Cormorbidity } \\
\hline Hypertension & $8(8.4)$ \\
\hline Diabetes mellitus & $5(5.3)$ \\
\hline Other diseases* & $7(7.4)$ \\
\hline None & 75 (78.9) \\
\hline \multicolumn{2}{|l|}{ Placement after discharge } \\
\hline Home & $67(70.5)$ \\
\hline Care facility & $22(23.2)$ \\
\hline Unknown & $6(6.3)$ \\
\hline
\end{tabular}

Table 2 Types of interventions during medication reconciliation ( $n=64$ cases)

\begin{tabular}{lc}
\hline Types of intervention & $\mathbf{n}(\%)$ \\
\hline Duration of prescription & $34(53.1)$ \\
Untreated indications & $18(28.1)$ \\
Drug selection & $5(7.8)$ \\
Duplication & $4(6.3)$ \\
Dose change & $1(1.6)$ \\
Drug interaction & $1(1.6)$ \\
Unintended omission & $1(1.6)$ \\
Total number of interventions & \\
\hline
\end{tabular}

*Intervention by physicians was accepted in $63(98 \%)$ of the 64 cases.

medications for treating the nervous system (32.2\%). The net acquisition cost saving for 5946 unused pills was US\$1711.35 (table 4). The potential avoidable cost with 64 interventions would be US\$ 2750.98 (0.3 X US\$143.28/day X 64 interventions) as US\$143.28 was the hospital room charge per day.

Based on the 2015 report by Healthcare Management Association in Korea, the average hospital pharmacists' salary in Korea was about US\$21.42/hour. ${ }^{10}$ In our study, pharmacists spent about 15 min reviewing a patient medical record before medication reconciliation and spent about $30 \mathrm{~min}$ performing medication reconciliation and other necessary interventions. Thus, the total time spent on the programme was about $45 \mathrm{~min}$ for each patient. Pharmacist labour cost was US $\$ 1349.46$ (US $\$ 21.42 /$ hour $\times 0.75$ hour $/$ pt $\times 56$ pts $\times 1.5$ pharmacists $)$. So it showed a clear cost-benefit with a net cost-benefit of US $\$ 3112.87$ (US\$1711.35+US\$2750.98-US\$1349.36) and a benefit: cost ratio of 2.31:1.
Table 3 Examples of interventions

\begin{tabular}{ll}
\hline Interventions & Intervention resolutions \\
\hline $\begin{array}{l}\text { Duplication of same mechanism medication } \\
\text { Granisetron patch and palonosetron IV }\end{array}$ & $\begin{array}{l}\text { Discontinue palonosetron } \\
\text { Discontinue cimetidine }\end{array}$ \\
$\begin{array}{l}\text { Omission of prescription } \\
\begin{array}{l}\text { Dexamethasone for prevention of } \\
\text { hypersensitivity and fluid retention with a } \\
\text { docetaxel regimen }\end{array}\end{array}$ & $\begin{array}{l}\text { Add dexamethasone } 8 \mathrm{mg} 3 \\
\text { times after chemotherapy }\end{array}$ \\
$\begin{array}{l}\text { Drug interaction } \\
\begin{array}{l}\text { Ciprofloxacin and domperidone, } \\
\text { granisetron and domperidone }\end{array}\end{array}$ \\
$\begin{array}{l}\text { Duration of prescription } \\
\begin{array}{l}\text { Prescribe a } 30 \text {-day prescription of lactulose } \\
\text { for constipation twice and continue } \\
\text { therapy until next admission }\end{array}\end{array}$ & Discontinue \\
\hline
\end{tabular}

Table 4 The amount and cost of unused medications upon admission $(n=95)$

\begin{tabular}{lcr}
\hline Therapeutic class & $\begin{array}{l}\text { Number of unused } \\
\text { pills, } \mathbf{n}(\%)\end{array}$ & $\begin{array}{c}\text { Cost } \\
\text { (US\$) }\end{array}$ \\
\hline A (Alimentary tract and metabolism) & $2819(47.4)$ & 599.03 \\
N (Nervous system) & $1912(32.2)$ & 575.96 \\
C (Cardiovascular system) & $154(2.6)$ & 63.37 \\
M (Musculoskeletal system) & $62(1.0)$ & 30.62 \\
J (Anti-infective agents for systemic use) & $20(0.3)$ & 10.37 \\
G (Genitourinary system and sex hormone) & $72(1.2)$ & 6.43 \\
D (Dermatological) & $32(0.5)$ & 4.47 \\
R (Respiratory system) & $75(1.3)$ & 4.40 \\
H (Systemic hormonal preparations) & 0 & 0 \\
B (Blood and blood-forming organs) & 0 & 0 \\
Others & $800(13.5)$ & 416.70 \\
Total & $5946(100.0)$ & 1711.35 \\
\hline
\end{tabular}

In March, the total number of pills returned was 19434 in the department, but after the programme implementation, 10132 pills were returned in May. The reduction rate was $47.9 \%$ (table 5). Cost saving by acquisition cost of unused drugs demonstrated a 52\% reduction between March (US\$3755) and May (US\$1968).

Of 95 patients, 58 patients $(61 \%)$ responded to our satisfaction survey of the medication reconciliation service. Among those 58, 55 patients (95\%) recognised the programme as a critical service for safe medication use. The other three patients stated that they did not need the service by pharmacist because they had sufficient knowledge to manage their medications as they had a long history of use. In addition, 30 of 31 responders $(97 \%)$ expressed their willingness to pay for a medication reconciliation service.

\section{DISCUSSION}

The amount of discarded medications increases every year. Healthcare costs and medication costs have also been rising. Medication waste is a financial burden, and also threatens environmental safety and public health. Experts found that overprescribing, frequent regimen changes, changes of medical condition and expiration of medications, all contribute to 
Table 5 Comparison of returned pill count and reduction rates at discharge in gynaecological oncology department between March and May

\begin{tabular}{|c|c|c|c|}
\hline \multirow[b]{2}{*}{ Therapeutic class } & \multicolumn{2}{|l|}{ Returned pills, n (\%) } & \multirow[b]{2}{*}{ Reduction rate $(\%$} \\
\hline & March (pre-programme) & May (post-programme) & \\
\hline A (Alimentary tract and metabolism) & $10837(55.8)$ & $6436(63.5)$ & 40.6 \\
\hline N (Nervous system) & $5782(29.8)$ & $2246(22.2)$ & 61.2 \\
\hline M (Musculoskeletal system) & $665(3.4)$ & $540(5.3)$ & 18.8 \\
\hline G (Genitourinary system and sex hormone) & $245(1.3)$ & $63(0.6)$ & 74.3 \\
\hline C (Cardiovascular system) & $100(0.5)$ & $14(0.1)$ & 86.0 \\
\hline J (Anti-infective agents for systemic use) & $60(0.3)$ & $161(1.6)$ & -168.3 \\
\hline B (Blood and blood-forming organs) & $43(0.2)$ & $74(0.7)$ & -72.1 \\
\hline H (Systemic hormonal preparations) & $1(0)$ & $110(1.1)$ & -10900.0 \\
\hline D (Dermatological) & $-(0.0)$ & $1(0.0)$ & - \\
\hline Others & $1701(8.8)$ & $487(4.8)$ & 71.4 \\
\hline Total & $19434(100)$ & $10132(100)$ & 47.9 \\
\hline
\end{tabular}

unnecessary medication waste. However, they indicated that pharmacy intervention programmes could prevent financial loss and unnecessary medication waste.

Previous studies in other countries on reduction of medication waste were conducted at primary care facilities and community pharmacies. $^{2} 11$ In Korea, the supply for discharge prescription medications ranges from 5 to 90 days, and our study shows that on average nine medications are filled at discharge. In order to assess the impact of medication reconciliation, we think it is important to target a patient population that requires routine visits. We found that the gynaecological oncology service was a good site at which to develop a medication reconciliation programme because most patients (1) have a scheduled admission every 3-4 weeks for chemotherapy, (2) have a high interest in health management, (3) are reliable and able to communicate. Patients in gynaecological oncology units are often considered as being in a non-acute care unit, and receive minor attention from the pharmaceutical care service, but we found that their usual hospital stay of 2-5 days was still sufficient to make effective interventions through medication reconciliation with the CMR.

In this prospective study, we assessed unused or expired medications as part of a medication reconciliation process. The quantity of unused or expired medications due to either non-compliance or overprescribing was calculated by pill counting and by self-report. Compliance can be measured by direct observation, symptomatic improvement, drug concentration in the blood or urine, pill counting and patient interview. Even though drug concentration levels may be the most accurate method of assessing compliance, it is not always feasible. Pill counting is a comparatively useful method, but it may not provide information on the level of drug intake or dose timing. Previous studies have shown that simply asking patients at the time of intervention is a successful method of obtaining an accurate measurement of compliance. In our study, we used both pill counting and patient interview to measure compliance.

Patients receiving chemotherapy are often readmitted with neutropenic fever or severe nausea, vomiting and diarrhoea shortly after discharge. To prevent readmission, emphasis should be placed on discharge counselling during transitions of care. We expected long-term care and nursing facilities to be in charge of refilling prescriptions based on medication changes, but we learnt during patient interviews that facilities focus on the administration of medication, and are seldom involved in refilling and dispensing medications. This indicated that pharmacists could play an important role in reducing unnecessary medications by dispensing a supply sufficient until the next scheduled hospital visit.

Medication reconciliation in our programme was performed in two steps. The first step included review of medical records, followed by patient interviews. This allowed pharmacists to perform a comprehensive review of the patient medical history, drug interactions and potential medication side effects due to prescribed medications and also to OTC medications, dietary supplements, self-administered medications and prescribed medications from different physicians. In Korea, patients prefers single-wrap-package dispensing, in which individual pills are dispensed together in one package for each dosing unit. This made it difficult to assess the expiry dates of pills which were brought from home. It was even harder with non-prescription and OTC medications. Alternative dispensing techniques need to be considered to prevent medication waste at the earlier step of the medication dispensing cycle. Furthermore, about $95 \%$ of patients agreed that medication reconciliation was valuable and critical in establishing a safe environment for drug administration in contrast to the traditional medication review programme. They understood the potential of the service to provide a positive influence on medication use.

Although the research reached its objectives, there were several limitations. Owing to the short study period, the sample size was small. Varkey et al discussed PDSA (Plan-Do-StudyAct), and found that patient medication reviews reduced the number of unnecessary prescriptions by at least $50 \%$ per patient, on average, and increased patient counselling sessions from $47.4 \%$ to $92.6 \% .^{12}$ They emphasised the usefulness of multidepartmental collaborations to establish a standardised medication process. In our programme, a close relationship among healthcare professionals in a gynaecological oncology unit, intellectual technical support and pharmacy services was the key factor enabling implementation of a successful medication reconciliation programme. This mutual collaboration was made possible by patient demand, readiness of departments to cooperate and interdisciplinary trust. Future studies should target a larger patient population in radiology, oncology, daysurgery, apheresis, or patients undergoing peritoneal dialysis.

According to McMullin et al, ${ }^{13}$ pharmacy review of patient medications reduced medication costs. One further limitation of our study is that only drug acquisition costs and the cost of pharmacist labour were directly calculated for the cost analysis. The cost of laboratory monitoring or treatment of adverse drug events was not available, which might have led to an 
underestimation of the cost saving. Another limitation includes the application of subjective probability values of 0.3 , according to Bates et al, as these probability values have not been validated. However, the cost savings reported in this study are conservative. Future studies considering other cost factors are necessary to further assess the impact of a medication reconciliation programme on the reduction of healthcare costs.

\section{CONCLUSIONS}

Our medication reconciliation service consisted of CMR and prevention of medication waste. It is imperative to widen these efforts to promote patient safety, reduce future healthcare costs and reduce drug waste.

\section{What this paper adds}

What is already known on this subject?

- Medication reconciliation is a key activity of the pharmaceutical care service.

- Medication reconciliation helps to improve treatment effectiveness and reduce medication errors.

- Medication reconciliation has been widely developed for outpatients receiving long-term care and for patients at acute and/or critical care units.

\section{What this study adds}

- Medication reconciliation may be beneficial for targeting patients with a scheduled hospital appointment who are aware of the importance of health self-management.

- Gynaecological oncology patients are good candidates for a medication reconciliation programme.

Acknowledgements We are grateful to Myungsook Min for supporting the program and Rose Kim for assisting with the preparation of the manuscript and its editing.

Contributors HYS and SJR participated in the research design. HYS, JMK, YML and SJR conducted the research. HYS and CK performed the data analysis. HYS, JJ,
CK and SJR wrote or contributed to writing of the manuscript. All authors read and approved the final manuscript.

Competing interests None declared.

Patient consent Obtained.

Ethics approval The institutional review board at Samsung Seoul Medical Center.

Provenance and peer review Not commissioned; externally peer reviewed.

\section{REFERENCES}

1 Karapinar-Carkt F, Borgsteede SD, Zoer J, et al. Effect of medication reconciliation with and without patient counseling on the number of pharmaceutical interventions among patients discharged from the hospital. Ann Pharmacother 2009:43:1001-10.

2 Nathan A, Goodyer L, Lovejoy A, et al. Brown bag medication reviews as a means of optimizing patients' use of medication and of identifying potential clinical problems. Fam Pract 1999;16:278-82.

3 U.S. Food and Drug Administration. Medication Disposal: Questions and Answers. Last updated: 03 March 2016. http://www.fda.gov/Drugs/ResourcesForYou/ Consumers/BuyingUsingMedicineSafely/EnsuringSafeUseofMedicine/ SafeDisposalofMedicines/ucm186188.htm (accessed 10 Sept 2016).

4 Chun P. Analysis of active pharmaceutical ingredients and drug cost of prescription medication returned to community pharmacies through drug-take back program. Yakhak Hoeji 2014;58:262-7.

5 Hazell B, Robson R. Pharmaceutical waste reduction in the National Health Service (NHS): a best practice compliance paper. version 1, 2015. https://www.england.nhs. uk/wp-content/uploads/2015/06/pharmaceutical-waste-reduction.pdf (accessed 2 Sept 2016).

6 Deffenbaugh J, ed. ASHP guidelines on a standardized method for pharmaceutical care. Best practices for health-system pharmacy. Bethesda, MD: American Society of Health System Pharmacists, 1996:109-11.

7 Bates DW, Cullen DJ, Laird N, et al. Incidence of adverse drug events and potential adverse drug events. Implications for prevention. ADE Prevention Study Group. J Am Med Assoc 1995;274:29-34.

8 Bates DW, Leape LL, Petrycki S. Incidence and preventability of adverse drug events in hospitalized adults. J Gen Intern Med 1993;8:289-94.

9 Kaushal R, Bates DW, Landrigan C, et al. Medication errors and adverse drug events in pediatric inpatients. J Am Med Assoc 2001;285:2114-20.

10 Korean Hospital Association and Korean Institute of Hospital Management. 2013 Hospital management statistics. http://www.kihm.re.kr/kxe (accessed 2 Sept 2016).

11 Andreoli L, Alexandra JF, Tesmoingt C, et al. Medication reconciliation: a prospective study in an internal medicine unit. Drugs Aging 2014:31:387-93.

12 Varkey P, Reller MK, Resar RK. Basics of quality improvement in health care. Mayo Clin Proc 2007:82:735-9.

13 McMullin ST, Hennenfent JA, Ritchie DJ, et al. A prospective, randomized trial to assess the cost impact of pharmacist-initiated interventions. Arch Intern Med 1999:159:2306-9. 